Article

\title{
Comparison of Illumina versus Nanopore 16S rRNA Gene Sequencing of the Human Nasal Microbiota
}

\author{
Astrid P. Heikema ${ }^{1, *}$, Deborah Horst-Kreft ${ }^{1}$, Stefan A. Boers ${ }^{2}$, Rick Jansen ${ }^{3}$, \\ Saskia D. Hiltemann ${ }^{3}$, Willem de Koning ${ }^{3}{ }^{\oplus}$, Robert Kraaij ${ }^{4}$, Maria A. J. de Ridder ${ }^{5}$, \\ Chantal B. van Houten ${ }^{6}$, Louis J. Bont ${ }^{6}$, Andrew P. Stubbs ${ }^{3}{ }^{\mathbb{D}}$ and John P. Hays ${ }^{1}$ \\ 1 Department of Medical Microbiology and Infectious Diseases, Erasmus University Medical Center \\ (Erasmus MC), 3015 CN Rotterdam, The Netherlands; d.kreft@erasmusmc.nl (D.H.-K.); \\ j.hays@erasmusmc.nl (J.P.H.) \\ 2 Department of Microbiology, Leiden University Medical Center (LUMC), 2333 ZA Leiden, The Netherlands; \\ s.a.boers@lumc.nl \\ 3 Department of Pathology, Erasmus University Medical Center (Erasmus MC), 3015 CN Rotterdam, \\ The Netherlands; r.jansen.1@erasmusmc.nl (R.J.); s.hiltemann@erasmusmc.nl (S.D.H.); \\ w.dekoning.1@erasmusmc.nl (W.d.K.); a.stubbs@erasmusmc.nl (A.P.S.) \\ 4 Department of Internal Medicine, Erasmus University Medical Center (Erasmus MC), 3015 CN Rotterdam, \\ The Netherlands; r.kraaij@erasmusmc.nl \\ 5 Department of Medical Informatics, Erasmus University Medical Center (Erasmus MC), 3015 CN Rotterdam, \\ The Netherlands; m.deridder@erasmusmc.nl \\ 6 Division of Paediatric Immunology and Infectious Diseases, University Medical Center Utrecht, \\ 3584 CX Utrecht, The Netherlands; c.b.vanhouten@umcutrecht.nl (C.B.v.H.); 1.bont@umcutrecht.nl (L.J.B.) \\ * Correspondence: a.heikema@erasmusmc.nl; Tel.: +31-107-033-510
}

Received: 29 August 2020; Accepted: 14 September 2020; Published: 21 September 2020

\begin{abstract}
Illumina and nanopore sequencing technologies are powerful tools that can be used to determine the bacterial composition of complex microbial communities. In this study, we compared nasal microbiota results at genus level using both Illumina and nanopore $16 \mathrm{~S}$ rRNA gene sequencing. We also monitored the progression of nanopore sequencing in the accurate identification of species, using pure, single species cultures, and evaluated the performance of the nanopore EPI2ME 16S data analysis pipeline. Fifty-nine nasal swabs were sequenced using Illumina MiSeq and Oxford Nanopore $16 \mathrm{~S}$ rRNA gene sequencing technologies. In addition, five pure cultures of relevant bacterial species were sequenced with the nanopore sequencing technology. The Illumina MiSeq sequence data were processed using bioinformatics modules present in the Mothur software package. Albacore and Guppy base calling, a workflow in nanopore EPI2ME (Oxford Nanopore Technologies-ONT, Oxford, UK) and an in-house developed bioinformatics script were used to analyze the nanopore data. At genus level, similar bacterial diversity profiles were found, and five main and established genera were identified by both platforms. However, probably due to mismatching of the nanopore sequence primers, the nanopore sequencing platform identified Corynebacterium in much lower abundance compared to Illumina sequencing. Further, when using default settings in the EPI2ME workflow, almost all sequence reads that seem to belong to the bacterial genus Dolosigranulum and a considerable part to the genus Haemophilus were only identified at family level. Nanopore sequencing of single species cultures demonstrated at least $88 \%$ accurate identification of the species at genus and species level for $4 / 5$ strains tested, including improvements in accurate sequence read identification when the basecaller Guppy and Albacore, and when flowcell versions R9.4 (Oxford Nanopore Technologies-ONT, Oxford, UK) and R9.2 (Oxford Nanopore Technologies-ONT, Oxford, UK) were compared. In conclusion, the current study shows that the nanopore sequencing platform is comparable with the Illumina platform in detection bacterial genera of the nasal microbiota, but the nanopore platform does have problems in detecting bacteria within the genus Corynebacterium. Although advances are being made, thorough validation of the nanopore platform is still recommendable.
\end{abstract}


Keywords: nasal microbiota; Illumina sequencing; nanopore sequencing; $16 \mathrm{~S}$ rRNA gene; bacterial species; Corynebacterium

\section{Introduction}

The use of traditional culture and established 16S rRNA gene sequencing techniques has shown that the composition of the nasal microbiota comprises microbiota profiles, dominated by four or five microbial genera. The microbiota composition varies in individuals with age [1], and shows large-scale variations in the first few years of life [2]. This variation usually involves colonization with Streptococcus pneumoniae, Haemophilus influenzae and Moraxella catarrhalis (three bacterial species often associated with the development of upper respiratory tract infections, including otitis media in young children) as well as Staphylococcus aureus, Dolosigranulum sp. or Corynebacterium spp. Further, the composition of the nasal microbiota has been associated with several other diseases, including the progression of cystic fibrosis [3], chronic rhinosinusitis [4], and progression to pneumonia after respiratory syncytial virus upper respiratory tract infection [5]. Nasal colonization with bacterial species such as Streptococcus pneumoniae, Haemophilus influenza, Moraxella catarrhalis, and Staphylococcus aureus may in the majority of cases be mutualistic or commensal, though a disturbance in this symbiotic relationship could lead to dysbiosis and disease, especially when these bacteria may also be present in the nasopharynx [6]. However, this phenomenon may not be related to microbiota profiles alone, but to a combination of bacterial, viral and child characteristics [7].

Unfortunately, traditional culture techniques are unable to detect a wide range of the so-called 'non-culturable' bacteria that DNA sequencing techniques have indicated to be present within the human nasal microbiota [8]. Also, to date, accurate species identification using 16S rRNA gene sequencing protocols in combination with the most popular sequencing platform (Illumina sequencing) is currently not universally possible as only short regions of bacterial $16 \mathrm{~S}$ rRNA genes tend to be sequenced using Illumina technology [9]. This means that the majority of microbiota publications to date have been limited to reporting the diversity of the (nasal) microbiota at best at the genus level. However, the accurate speciation of bacteria can be very important for clinicians as a bacterial genus may contain several species that possess very different virulence characteristics [10]. For example, being able to differentiate between a Staphylococcus aureus and a Staphylococcus epidermidis infection may be significant in the treatment of sepsis or skin infections.

Nanopore sequencing (Oxford Nanopore Technologies-ONT, Oxford, UK) [11], is a 'third generation' (i.e., single-molecule) sequencing technology that is able to generate long sequence read-lengths that can span the majority of the bacterial 16S rRNA gene. Several recent comparative studies demonstrated promising results for the nanopore technology including identification of the microbiota composition at the species level. For example, a significantly similar bacterial composition at genus level and the identification of more bacterial species was reported when Oxford Nanopore and Illumina 16S rRNA gene sequencing were compared for the mouse gut microbiota [12]. In another study, the performance of nanopore versus IonTorrent $P G M^{\circledR}$ sequencing on mock and dog skin microbiota samples indicated increased bacterial richness at high taxonomic levels (species identification) associated with nanopore sequencing [13]. In a separate time course analysis, nanopore $16 \mathrm{~S}$ rRNA gene sequencing resulted in the detection of all 20 of the bacterial species present in a mock bacterial community within minutes [14]. A drawback of nanopore sequencing is the relatively high sequencing error rate, ranging from 5\% [1] to $38.2 \%$ [15]. This further complicates accurate taxonomy at species level, particularly for bacterial species with a high sequence similarity in the 16S RNA gene.

Although comparisons of nanopore sequencing with other sequencing systems have previously been published, to our knowledge no comparative data were published with a specific focus on the nasal microbiota. The nasal microbiota contains microbial species at lower microbial abundance compared to high-biomass samples such as feces. It, however, may be a source of potential antibiotic-resistant 
pathogens such as methicillin-resistant Staphylococcus aureus (MRSA) [16]. In this manuscript, we compared Illumina versus nanopore sequencing at genus level using nose swab samples that had been obtained from the European Union-funded FP7 project [17]. Initial comparative research was performed using version R9.2 nanopore sequencing devices (flowcells), the Albacore basecaller and earlier versions of the EPI2ME $16 \mathrm{~S}$ sequence data analysis pipeline, which is still evolving and being updated by ONT [18]. Therefore, subsequent to, and based on, the results of our initial comparative analysis, we performed further analysis and investigated the potential effect of newer ONT advancements (EPI2ME, the Guppy basecaller and flowcells R9.4) on the results of microbiota profiling at genus and species level using pure cultures of relevant bacterial species.

\section{Material and Methods}

\subsection{Sample Collection and Selection}

Fifty-nine nose swab samples generating at least 1000 Illumina sequence reads and $3 \times 10^{3} 16 \mathrm{~S}$ rRNA gene copies per microliter were randomly selected for nanopore $16 \mathrm{~S}$ rRNA gene sequencing. These samples had been previously obtained from patients with lower respiratory tract infections, sepsis, and non-infected control patients participating in the EU FP7-funded TAILORED-treatment study, and Illumina sequenced. They comprised nose swab samples from 10 adults and 49 children under the age of 18 . Seven negative control swabs were also sequenced, containing nasal swab Universal Transport Medium (UTM, ESwab ${ }^{\mathrm{TM}}$, COPAN Diagnostics Inc., Brescia, Italy) only.

\subsection{DNA Isolation}

DNA was previously isolated from nasal swab samples using the mag mini kit (LGC Standards, Wesel, Germany) and an adjusted protocol that included an initial bead-beating step. In short, $200 \mu \mathrm{L}$ of nose swab medium combined with $200 \mu \mathrm{L}$ phenol and $150 \mu \mathrm{L}$ Lysis buffer BL (LGC Standards, Wesel, Germany) was added to a vial containing Lysing Matrix beads (MP Biomedicals, Eschwege, Germany) and subjected to bead-beating using a FastPrep-24 (MP Biomedicals, Eschwege, Germany) at $6 \mathrm{~m} / \mathrm{s}$ for $60 \mathrm{~s}$. After centrifugation, $200 \mu \mathrm{L}$ of the water phase (top layer) was incubated for $2 \mathrm{~min}$ at room temperature with $400 \mu \mathrm{L}$ binding buffer BL (LGC Standards, Wesel, Germany), to which $10 \mu \mathrm{L}$ mag particle suspension (LGC Standards, Wesel, Germany) had been added. The manufacturer's protocol was then followed, with the exception that the DNA was eluted by incubating for $30 \mathrm{~min}$ at $55^{\circ} \mathrm{C}$ instead of $10 \mathrm{~min}$. Prior to $16 \mathrm{~S}$ rRNA gene sequencing, the total number of $16 \mathrm{~S}$ rRNA gene copy numbers within each DNA extract was measured using a 16S rRNA gene quantitative PCR as previously described [19].

\subsection{Bacterial Strains}

The following purely cultured bacterial strains were used in this study: Haemophilus influenzea ATCC 10211, Moraxella catarrhalis ATCC 25240, Staphylococcus aureus ATCC 25923, Staphylococcus epidermidis ATCC 12228, Streptococcus pneumoniae ATCC 49619, Corynebacterium diphtheria ATCC 13812, and from our own hospital strain collection: Corynebacterium accollens, Corynebacterium amycolatum, Corynebacterium pseudodiphtheriticum, and Corynebacterium striatum. The identity of the hospital isolates used was confirmed by matrix-assisted laser desorption ionization-time of flight spectrometry (MALDI-TOF MS, Bruker Daltonics).

\subsection{Illumina Sequencing}

The hypervariable V5 and V6 regions (276 base pairs-bp) of the 16S rRNA gene were amplified using the 785F (5'-GGA TTA GAT ACC CBR GTA GTC-3') and 1061R (5'-TCA CGR CAC GAG CTG ACG AC-3') primers [20], and dual indexing [21]. Amplicons were generated in 30 cycli using the FastStart High Fidelity System (Roche, Woerden, The Netherlands), normalized using the SequalPrep Normalization Plate kit (Thermo Fischer Scientific, Breda, The Netherlands) and pooled in batches of 
approximately 250 samples. Pools were purified prior to sequencing using the Agencourt AMPure XP (Beckman Coulter Life Science, Indianapolis, IN, USA), and the amplicon size and quantity of the pools were assessed on the LabChip GX (PerkinElmer Inc., Groningen, The Netherlands). The PhiX Control v3 library (Illumina Inc., San Diego, CA, USA) was combined ( 10\%) with the pooled amplicon libraries and each pool was sequenced on an Illumina MiSeq sequencer (MiSeq Reagent Kit v3, $2 \times 300$ bp).

\subsection{Nanopore Sequencing}

$16 \mathrm{~S}$ rRNA gene sequence libraries were prepared with the 16S Rapid Amplicon Barcoding Kit (Oxford Nanopore Technologies-ONT, Oxford, UK, SQK-RAB201) according to the standard procedures described by ONT. The complete $16 \mathrm{~S}$ rRNA gene was amplified using $10 \mu \mathrm{L}$ input DNA purified from nasal swabs, LongAmp ${ }^{\circledR}$ Taq $2 \times$ master mix (New England Biolabs, Ipswich, MA, USA) and the barcoded nanopore sequence primers 27F 5'-AGA GTT TGA TCM TGG CTC AG-3' and 149R 5'-CGG TTA CCT TGT TAC GAC TT-3'. The DNA amplification was performed on a T100 Thermal Cycler (Biorad, Lunteren, The Netherlands) using the program; 1 min denaturation at $95{ }^{\circ} \mathrm{C}, 25$ cycles $\left(95^{\circ} \mathrm{C}-20 \mathrm{~s}, 55^{\circ} \mathrm{C}-30 \mathrm{~s}, 68^{\circ} \mathrm{C}-2 \mathrm{~min}\right)$ and a final extension step of $5 \mathrm{~min}$ at $65^{\circ} \mathrm{C}$. The $16 \mathrm{~S}$ rRNA gene amplicons were quantified using Quant-IT ${ }^{\mathrm{TM}}$ PicoGreen $^{\mathrm{TM}}$ (Thermo Fisher Scientific, Breda, The Netherlands), equal amounts of amplicons per sample were pooled and the library was further processed as described by the manufacturer. Next, the library was incubated with Library Loading Beads (Oxford Nanopore Technologies-ONT, Oxford, UK) and the mixture was added to the MinIon/GridIon flow cell (version R9.2 or R.9.4, Oxford Nanopore Technologies-ONT, Oxford, UK). Sequencing was performed using a MinIon or GridIon nanopore sequencer (Oxford Nanopore Technologies-ONT, Oxford, UK) for approximately $16 \mathrm{~h}$.

\subsection{Data Analysis}

The Illumina MiSeq sequence data were analyzed using bioinformatics modules present in the Mothur software package [22], that we previously integrated into Galaxy (i.e., Galaxy mothur Toolset, Gm [23]). In short, forward and reverse FASTQ-formatted sequence files were merged using the make.contigs command. Primer sequences were trimmed and sequences that had an ambiguous base call (N) in the sequence or with lengths smaller than 200 were removed from the analysis. Unique sequences were then aligned against a customized reference alignment based on the SILVA reference alignment release $123[24,25]$. The reference sequences were trimmed to only include the V5-V6 region of the $16 \mathrm{~S}$ rRNA gene using the pcr.seqs command. Sequences that did not align to this region were culled from further analysis and the alignments were trimmed so that the sequences fully overlapped the same alignment coordinates. Next, sequences were further de-noised by pre-clustering the sequences using the pre.cluster command allowing for up to two differences between sequences, and potentially chimeric sequences were removed using Uchime, as implemented in Mothur. The remaining sequences were classified using the classify.seqs command with the customized SILVA alignment release 123 as reference. Finally, sequences were clustered into operational taxonomic units (OTUs) at $97 \%$ similarity using the default settings of the dist.seq and cluster commands respectively, and the classify.otu algorithm was used to get a consensus taxonomy for each OTU.

Basecalling of nanopore signals was performed using the MinKNOW (MinION software, version 1.6, Oxford Nanopore Technologies-ONT, Oxford, UK) embedded Albacore version 1.0 data processing pipeline or the Guppy version 3.2.10 pipeline (Oxford Nanopore Technologies-ONT, Oxford, UK). The Barcoding workflow in the Metrichor Ltd. analysis platform EPI2ME (Oxford Nanopore Technologies-ONT, Oxford, UK) [26] was used for the de-barcoding of the sequence reads derived from the nose swab samples sequenced with the Oxford Nanopore platform. For the identification of bacteria at genes and species level, fast 5 or fastq files containing full length 16S rRNA gene amplicons where uploaded to the EPI2ME desktop agent 16S workflow (versions 2.47.53720F8, 2.48.690655 or 2020.2.10, Oxford Nanopore Technologies-ONT, Oxford, UK) where each file was classified real-time using the NCBI 16S rRNA gene blast database [27]. Blastn was run using the 
parameters max_target seqs $=3$ (finds the top three hits that are statistically significant) and output $\mathrm{fmt}=6$. The number of genera represented in the top three classifications (num_genus_taxid) was calculated along with the genus rank (if classified at genus rank or below) per sequencing record. These were calculated using the Python library ete2 [28], which utilizes the NCBI taxonomy. The top scoring classification per individual record within the file was selected as the read classification along with the accompanying num_genus_taxid and genus and species information. Coverage information per read was calculated as number of identical matches/query length. All read classifications were then filtered for $>77 \%$ accuracy and $>30 \%$ coverage, which removes spurious alignments. Results were returned via a web report and can be downloaded as a comma-separated values (CSV) file.

Then, the results in the CSV file of the EPI2ME 16S workflow output were used for further analysis using an in-house-generated Python script together with the Python ete2 package. This script reads the contents of the CSV file and retrieves the species and genus names from the NCBI taxonomy IDs found by the EPI2ME 16S workflow. Exclusion criteria for the single nanopore reads were an alignment count accuracy $<80 \%$, quality score (QC) score $<7$, read length $<1400>1700 \mathrm{bp}$, and a num_genus_taxid other than 1 or 2 . These exclusion criteria apply for the initial analyses of the nasal swab samples in this study. For the nasal swab samples that were re-basecalled with Guppy, and the purely cultured bacterial strains that were (re-) basecalled with Guppy, the applied exclusion criteria were: alignment count accuracy $85 \%$, QC score $<9$, read length $<1400>1700 \mathrm{bp}$, and an lca score other than 0 . For species level identification, similar criteria and the highest scoring BLAST identification (top rank) was used. The higher accuracy and QC thresholds were chosen because (re-) basecalling with Guppy or using a R.9.4 flowcell resulted in a higher average QC score (from at least 7 to $\sim 10$ ) and accuracy (from $~ 85 \%$ to $~ 90 \%$ ) in the EP2ME analysis (R9.2 flowcell, Albacore basecalling versus R9.2 or R9.4 flowcell and Guppy basecalling, respectively, data not shown). On average, $15 \%$ of the reads were excluded after re-basecalling with Guppy and filtering with the more stringent thresholds (data not shown).

\subsection{Statistics}

Rarefaction analysis was performed to determine the amount of reads needed to accurately assess the bacteria richness in the samples (Supplementary Figure S1). Plots were generated with QIIME (Quantitative Insights Into Microbial Ecology) version 1.9.1 (multiple_rarefactions.py, alpha_diversity.py, collate_alpha.py, make_rarefaction_plots.py) using the Shannon diversity metric. Based on the rarefraction analysis, samples generating $>500$ sequence reads were included for bioinformatics analysis.

Taxonomy results of the data produced after Illumina and nanopore sequencing were loaded into BioNumerics software version 7.6 (Applied Math, Sint-Martens-Latem, Belgium) and a phylogenetic tree was generated based on the relative abundance proportions of the genera (normalized to $100 \%$ ), the Pearson's correlation coefficient and the UPGMA algorithm. Microbiota profiles generated after Illumina or nanopore sequencing were visualized using Microsoft Excel 2010, and ordered based on the sample order in the phylogenetic tree. Alpha-diversity at the genus level was assessed using two metrics: the number of observed genera present with an abundance of at least $1 \%$, and the inverse Simpson index (ISI). Bland-Altman plots were made to explore the comparability of the microbiota profiles generated by Illumina and nanopore sequencing for the six most prevalent genera. These plots show the difference in measured percentages between the two methods versus the mean of the measured percentages.

\subsection{Sequence Data Availability}

The Illumina and nanopore sequence datasets of the nose swab samples, generated and analyzed in the current study, are available in the European Nucleotide Archive (ENA) under accession number PRJEB28612 [29]. 


\section{Results}

\subsection{Sample Population}

Fifty-one nose swab samples from patients with a respiratory tract infection or sepsis and eight control patients (no infection) were included in the study (Table 1). Most patients were children under the age of 5 years $(37 / 59,63 \%)$. It should be noted that the current analysis was designed to investigate differences between Illumina and nanopore sequencing of nasal microbiota profiles and not to determine possible differences between infection versus no-infection or children versus adult patient populations.

\subsection{General Sequencing Results}

An average of 131,024 raw reads were generated per sample using the Illumina MiSeq platform, with a mean of $91 \%$ of raw reads being classified into a mean of 4.4 genera, which were present with an abundance of $\geq 1 \%$ per sample (Table 1 ). Using nanopore sequencing, an average of 21,907 raw reads were obtained per sample and a mean of $78 \%$ of the raw reads were classified into a mean of 4.5 genera, which were present with an abundance of $\geq 1 \%$ per sample (Table 1 ). The Illumina platform resulted in a significantly higher ISI compared to nanopore; 2.7 vs. 2.2, $p<0.0001$, paired T. test (Table 1).

For the data generated using nanopore sequencing, 2/59 (3.4\%) of the samples were below the cut-off of 500 reads. These samples were excluded from further analysis. Low read numbers ranging from 1-3408 reads for the Illumina platform and 0-56 reads for nanopore were detected in negative control samples $(n=7)$.

\subsection{Illumina versus Nanopore Sequencing}

Phylogenetic clustering of the taxonomy results (normalized to $100 \%$ ) generated after Illumina sequencing provided five microbial clades (I-V, Figure 1a). Clade I was dominated by Moraxella spp.; II had a mixture of Moraxella spp., Dolosigranulum sp. and Corynebacterium spp.; III Dolosigranulum sp. and Corynebacterium spp.; IV Haemophilus spp.; and V Staphylococcus spp. (Figure 1a). When using the Illumina platform, Corynebacterium spp., Moraxella spp., Dolosigranulum sp., and Streptococcus spp. were most prevalent, and $1 \%$ or more of these genera could be detected in $46,44,43$, and 32 of the 57 samples analyzed, respectively. 
Table 1. Nose swab samples of individuals and negative controls that were sequenced using and Illumina and nanopore 16S rRNA gene sequencing technologies. (a) = a maximum of 5000 raw Illumina sequence reads were analyzed for the classification of genera. ${ }^{\text {(b) }}=$ samples with read numbers below the 500 read cut-off. NA = not applicable.

\begin{tabular}{|c|c|c|c|c|c|c|c|c|c|c|c|}
\hline \multicolumn{4}{|c|}{ Sample Information } & \multicolumn{4}{|c|}{ Illumina Technology } & \multicolumn{4}{|c|}{ Nanopore Technology } \\
\hline Sample & Infection & $\begin{array}{c}\text { Age } \\
\text { (Years) }\end{array}$ & 16S Copies & $\begin{array}{c}\text { Raw } \\
\text { Reads }\end{array}$ & $\begin{array}{l}\text { Percentage Reads } \\
\text { Classified (\%) (a) }\end{array}$ & $\begin{array}{l}\text { Genera } \\
\text { Identified } \\
\geq \mathbf{1} \%\end{array}$ & $\begin{array}{c}\text { Genera } \\
\text { (ISI) }\end{array}$ & $\begin{array}{l}\text { Raw } \\
\text { Reads }\end{array}$ & $\begin{array}{c}\text { Percentage } \\
\text { Reads } \\
\text { Classified (\%) }\end{array}$ & $\begin{array}{c}\text { Genera } \\
\text { Identified } \\
\geq \mathbf{1} \%\end{array}$ & $\begin{array}{c}\text { Genera } \\
\text { (ISI) }\end{array}$ \\
\hline 1 & yes & 3.50 & $5 \times 10^{5}$ & 133,880 & 92 & 5 & 4.2 & 34,944 & 77 & 5 & 3.0 \\
\hline 2 & yes & 0.92 & $1 \times 10^{5}$ & 186,250 & 95 & 5 & 1.9 & 15,254 & 79 & 3 & 2.2 \\
\hline 3 & yes & 2.00 & $3 \times 10^{5}$ & 1661 & 94 & 5 & 4.1 & 39,474 & 77 & 4 & 2.9 \\
\hline 4 & yes & 1.50 & $3 \times 10^{5}$ & 154,877 & 96 & 7 & 4.6 & 36,608 & 76 & 6 & 2.3 \\
\hline 5 & yes & 9.00 & $3 \times 10^{5}$ & 114,702 & 97 & 5 & 3.5 & 5107 & 59 & 4 & 2.7 \\
\hline 6 & yes & 2.00 & $3 \times 10^{5}$ & 22,805 & 97 & 5 & 2.7 & 31,642 & 52 & 4 & 1.7 \\
\hline 7 & yes & 5.00 & $2 \times 10^{5}$ & 1940 & 88 & 8 & 3.8 & 2246 & 57 & 6 & 3.1 \\
\hline 8 & yes & 4.00 & $3 \times 10^{5}$ & 24,214 & 100 & 4 & 1.2 & 10,174 & 62 & 3 & 1.2 \\
\hline 9 & yes & 1.67 & $4 \times 10^{5}$ & 104,134 & 93 & 9 & 2.5 & 21,462 & 68 & 6 & 2.6 \\
\hline 10 & yes & 8.00 & $2 \times 10^{5}$ & 186,945 & 96 & 3 & 2.5 & 923 & 68 & 2 & 1.6 \\
\hline 11 & yes & 11.00 & $2 \times 10^{5}$ & 120,867 & 95 & 3 & 3.0 & 27,569 & 78 & 3 & 1.6 \\
\hline 12 & yes & 0.42 & $4 \times 10^{5}$ & 25,743 & 98 & 3 & 3.0 & 5127 & 66 & 3 & 2.2 \\
\hline 13 & yes & 15.00 & $4 \times 10^{5}$ & 261,123 & 95 & 4 & 2.7 & 12,572 & 66 & 5 & 2.0 \\
\hline 14 & yes & 2.17 & $1 \times 10^{5}$ & 6246 & 97 & 4 & 3.0 & 20,441 & 89 & 3 & 2.7 \\
\hline 15 & yes & 3.80 & $3 \times 10^{5}$ & 68,095 & 91 & 3 & 2.3 & 27,077 & 90 & 4 & 2.5 \\
\hline 16 & yes & 2.40 & $1 \times 10^{5}$ & 119,295 & 84 & 7 & 2.9 & 2978 & 85 & 6 & 2.6 \\
\hline 17 & yes & 0.80 & $2 \times 10^{5}$ & 74,902 & 96 & 3 & 1.5 & 4408 & 91 & 2 & 1.1 \\
\hline 18 & yes & 61.00 & $3 \times 10^{3}$ & 77,851 & 86 & 6 & 3.4 & 2141 & 82 & 8 & 4.1 \\
\hline 19 & yes & 0.90 & $3 \times 10^{5}$ & 74,730 & 85 & 4 & 2.3 & 20,584 & 82 & 6 & 1.6 \\
\hline 20 & yes & 0.80 & $3 \times 10^{5}$ & 113,078 & 93 & 3 & 2.4 & 10,974 & 91 & 3 & 1.9 \\
\hline 21 & yes & 78.00 & $2 \times 10^{5}$ & 131,837 & 90 & 2 & 1.7 & 21,449 & 93 & 1 & 1.0 \\
\hline 22 & yes & 1.70 & $3 \times 10^{5}$ & 162,890 & 85 & 4 & 2.4 & 23,530 & 92 & 5 & 1.8 \\
\hline 23 & yes & 2.30 & $2 \times 10^{5}$ & 83,596 & 92 & 8 & 4.4 & 15,748 & 88 & 7 & 3.2 \\
\hline 24 & yes & 73.00 & $2 \times 10^{5}$ & 83,947 & 84 & 4 & 2.0 & 3181 & 88 & 5 & 3.3 \\
\hline 25 & yes & 2.60 & $5 \times 10^{5}$ & 28,221 & 92 & 3 & 3.0 & 15,453 & 50 & 3 & 3.1 \\
\hline 26 & yes & 65.00 & $3 \times 10^{5}$ & 77,012 & 82 & 7 & 4.5 & 31,461 & 85 & 6 & 2.8 \\
\hline 27 & yes & 0.80 & $1 \times 10^{5}$ & 58,962 & 85 & 3 & 2.5 & 23,652 & 90 & 3 & 1.5 \\
\hline 28 & yes & 3.00 & $5 \times 10^{5}$ & 57,600 & 86 & 6 & 3.7 & 22,991 & 84 & 7 & 3.4 \\
\hline 29 & yes & 57.00 & $2 \times 10^{5}$ & 129,131 & 94 & 2 & 1.5 & 48,167 & 90 & 1 & 1.1 \\
\hline 30 & yes & 0.40 & $6 \times 10^{5}$ & 180,796 & 88 & 3 & 2.9 & 3997 & 65 & 4 & 2.3 \\
\hline 31 & yes & 0.90 & $4 \times 10^{5}$ & 547,695 & 98 & 4 & 2.7 & 15,626 & 80 & 7 & 1.7 \\
\hline 32 & yes & 23.00 & $8 \times 10^{5}$ & 750,669 & 97 & 3 & 1.8 & 6653 & 67 & 2 & 1.6 \\
\hline 33 & yes & 3.40 & $1 \times 10^{5}$ & 924,890 & 98 & 7 & 3.3 & 25,148 & 74 & 7 & 2.1 \\
\hline 34 & yes & 4.10 & $1 \times 10^{6}$ & 31,896 & 94 & 5 & 4.0 & 15,979 & 49 & 4 & 2.7 \\
\hline 35 & yes & 14.00 & $3 \times 10^{5}$ & 79,970 & 90 & 3 & 2.1 & 40,551 & 88 & 3 & 1.4 \\
\hline 36 & yes & 0.10 & $3 \times 10^{5}$ & 113,047 & 88 & 3 & 1.7 & 50 & 76 & NA & NA \\
\hline $37^{\text {(b) }}$ & yes & 0.40 & $3 \times 10^{5}$ & 59,397 & 88 & 4 & 2.9 & 51,254 & 63 & 11 & 3.6 \\
\hline 38 & yes & 0.30 & $6 \times 10^{5}$ & 7421 & 99 & 3 & 1.4 & 41,757 & 89 & 2 & 1.4 \\
\hline
\end{tabular}


Table 1. Cont.

\begin{tabular}{|c|c|c|c|c|c|c|c|c|c|c|c|}
\hline \multicolumn{4}{|c|}{ Sample Information } & \multicolumn{4}{|c|}{ Illumina Technology } & \multicolumn{4}{|c|}{ Nanopore Technology } \\
\hline Sample & Infection & $\begin{array}{c}\text { Age } \\
\text { (Years) }\end{array}$ & 16S Copies & $\begin{array}{c}\text { Raw } \\
\text { Reads }\end{array}$ & $\begin{array}{l}\text { Percentage Reads } \\
\text { Classified (\%) (a) }\end{array}$ & $\begin{array}{c}\text { Genera } \\
\text { Identified } \\
\geq \mathbf{1} \%\end{array}$ & $\begin{array}{l}\text { Genera } \\
\text { (ISI) }\end{array}$ & $\begin{array}{l}\text { Raw } \\
\text { Reads }\end{array}$ & $\begin{array}{l}\text { Percentage } \\
\text { Reads } \\
\text { Classified (\%) }\end{array}$ & $\begin{array}{c}\text { Genera } \\
\text { Identified } \\
\geq \mathbf{1} \%\end{array}$ & $\begin{array}{l}\text { Genera } \\
\text { (ISI) }\end{array}$ \\
\hline 39 & yes & 1.10 & $3 \times 10^{5}$ & 121,819 & 86 & 3 & 2.6 & 6340 & 86 & 6 & 1.9 \\
\hline 40 & yes & 0.20 & $2 \times 10^{5}$ & 83,457 & 83 & 4 & 2.4 & 59,923 & 82 & 6 & 1.9 \\
\hline 41 & yes & 4.20 & $4 \times 10^{5}$ & 92,006 & 87 & 4 & 2.9 & 17,785 & 90 & 4 & 2.3 \\
\hline 42 & yes & 0.10 & $1 \times 10^{5}$ & 36,248 & 90 & 4 & 2.0 & 45,047 & 92 & 3 & 1.9 \\
\hline 43 & yes & 0.10 & $2 \times 10^{5}$ & 55,585 & 92 & 5 & 2.3 & 47,084 & 92 & 3 & 1.4 \\
\hline 44 & yes & 0.40 & $3 \times 10^{5}$ & 101,465 & 87 & 5 & 2.7 & 5288 & 80 & 6 & 1.6 \\
\hline 45 & yes & 1.70 & $7 \times 10^{5}$ & 92,476 & 89 & 3 & 1.9 & 49,104 & 55 & 2 & 1.1 \\
\hline 46 & yes & 0.50 & $3 \times 10^{5}$ & 72,068 & 88 & 4 & 2.1 & 50,486 & 80 & 6 & 1.5 \\
\hline 47 & yes & 0.10 & $5 \times 10^{5}$ & 90,128 & 80 & 6 & 4.0 & 107,161 & 91 & 6 & 3.2 \\
\hline $48^{(\mathrm{b})}$ & yes & 67.00 & $2 \times 10^{5}$ & 51,826 & 94 & 5 & 1.3 & 8 & 75 & NA & NA \\
\hline 49 & yes & 0.30 & $9 \times 10^{5}$ & 1148 & 82 & 8 & 4.3 & 14,673 & 66 & 3 & 1.5 \\
\hline 50 & yes & 3.30 & $5 \times 10^{6}$ & 39,030 & 83 & 3 & 2.6 & 12,239 & 66 & 3 & 2.0 \\
\hline 51 & yes & 56.00 & $5 \times 10^{6}$ & 2191 & 85 & 7 & 3.3 & 17,248 & 64 & 7 & 2.4 \\
\hline 52 & no & 28.00 & $3 \times 10^{5}$ & 193,859 & 96 & 2 & 1.2 & 6,789 & 91 & 1 & 1.0 \\
\hline 53 & no & 62.00 & $2 \times 10^{5}$ & 262,184 & 89 & 3 & 2.3 & 18,680 & 88 & 2 & 1.8 \\
\hline 54 & no & 8.10 & $2 \times 10^{5}$ & 308,123 & 83 & 5 & 2.8 & 13,741 & 89 & 4 & 2.3 \\
\hline 55 & no & 7.20 & $3 \times 10^{5}$ & 203,242 & 100 & 6 & 3.4 & 15,490 & 84 & 6 & 4.3 \\
\hline 56 & no & 14.90 & $1 \times 10^{5}$ & 235,820 & 92 & 3 & 1.4 & 18,318 & 88 & 8 & 5.0 \\
\hline 57 & no & 5.40 & $9 \times 10^{5}$ & 90,422 & 86 & 3 & 2.8 & 11,207 & 87 & 4 & 2.2 \\
\hline 58 & no & 7.10 & $6 \times 10^{5}$ & 103,176 & 87 & 5 & 2.9 & 19,604 & 73 & 7 & 2.7 \\
\hline 59 & no & 6.40 & $1 \times 10^{5}$ & 111,844 & 93 & 4 & 1.6 & 17,971 & 88 & 3 & 1.1 \\
\hline Average & NA & 12.5 & $8 \times 10^{5}$ & 131,024 & 91 & 4.4 & 2.7 & 21,907 & 78 & 4.5 & 2.2 \\
\hline \multicolumn{12}{|l|}{ Control } \\
\hline $\mathrm{C}-1$ & NA & NA & $<1 \times 10^{2}$ & 6 & 0 & 0 & NA & 7 & 57 & 4 & NA \\
\hline C- 2 & NA & NA & $<1 \times 10^{2}$ & 1 & 0 & 0 & 0 & 42 & 74 & 8 & NA \\
\hline C-3 & NA & NA & $<1 \times 10^{2}$ & 1 & 0 & 0 & 0 & 33 & 42 & 9 & NA \\
\hline C-4 & NA & NA & $<1 \times 10^{2}$ & 3 & 0 & 0 & 0 & 35 & 51 & 11 & NA \\
\hline C-5 & NA & NA & $<1 \times 10^{2}$ & 2 & 0 & 0 & 0 & 15 & 67 & 3 & NA \\
\hline$C-6$ & NA & NA & $2 \times 10^{2}$ & 2440 & 98 & 4 & 4 & 56 & 91 & 6 & NA \\
\hline C-7 & NA & NA & $3 \times 10^{2}$ & 3408 & 94 & 18 & 18 & 0 & 0 & 0 & NA \\
\hline
\end{tabular}




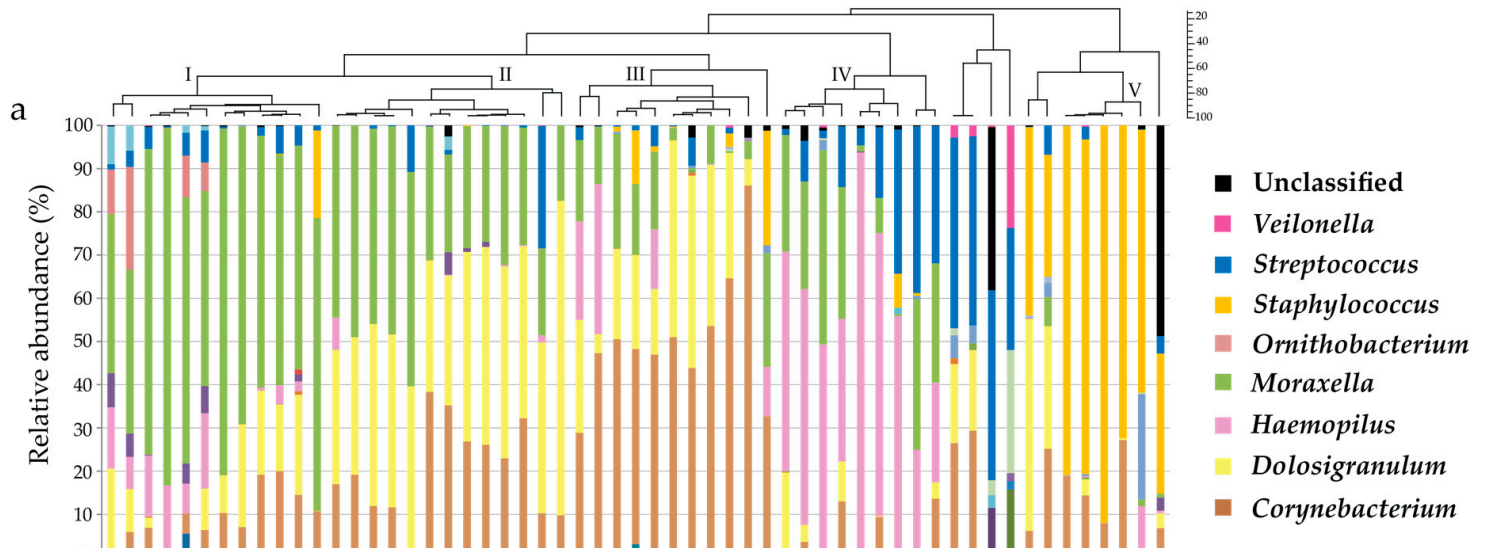

b

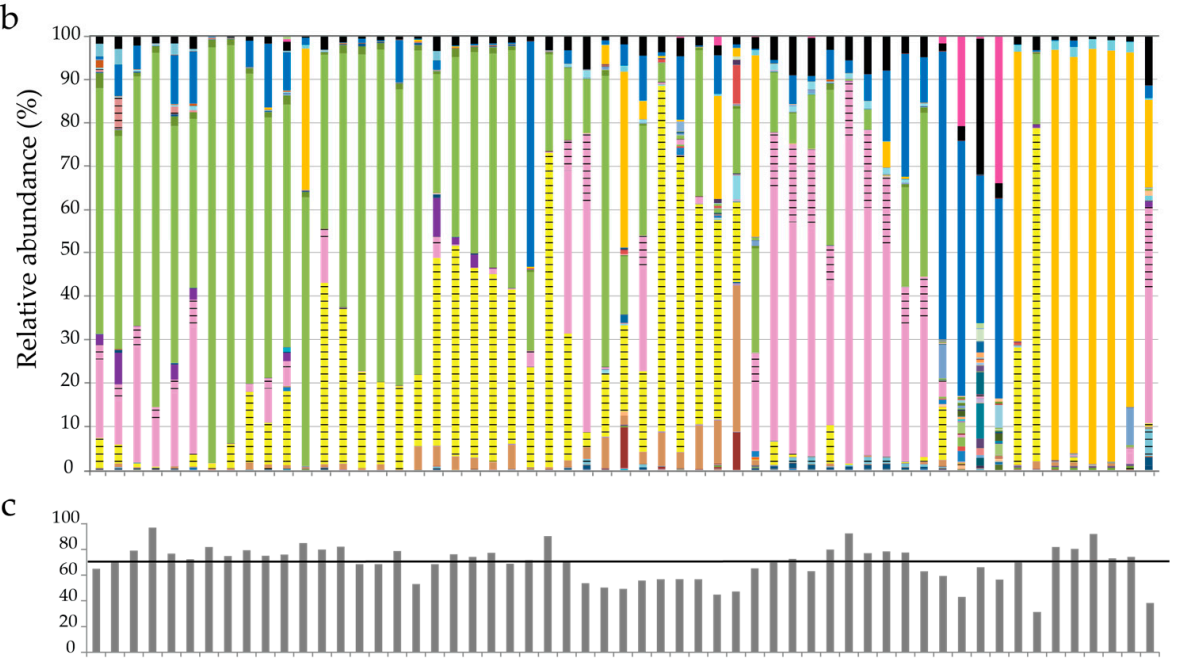

Figure 1. Nasal microbiota profiles generated using nanopore and Illumina 16S rRNA gene sequencing. DNA was isolated from 57 nose swab samples, and 16S rRNA gene sequencing was performed using both Illumina (a) and nanopore (b) technologies. Each bar in the graph represents a nasal microbiota profile from a single individual. The dashed lines in (b) represent genera that, by default, were reported as unclassified at genus level in the EPI2ME report but were identified when next to reads with a top three blast hit with one genera (num_genus_taxid is 1); reads with a top three blast hit with two genera (num_genus_taxid is 2) were also included. A phylogenetic tree was generated by Pearson/UPGMA clustering of bacterial genera in microbiota profiles, as determined using Illumina sequencing. To compare between the two techniques, the sample order of the samples that were sequenced with the Oxford Nanopore platform was matched to the sample order of the samples that were sequenced with the Illumina platform, and the percentage of agreement was calculated for each nose swab sample (c). The horizontal black line in (c) indicates the mean percentage of agreement.

In general, a similar microbiota composition was observed when the genus taxonomy results derived from the two sequencing methods, Illumina and nanopore, were aligned and compared (Figure 1a,b). However, initially, in the nanopore sequenced samples, Dolosigranulum sp. was classified in very low abundance (none of that samples had $>1 \%$ ) in the EPI2ME output. By default, the EPI2ME report (EPI2ME version 2.47.537208 and 2.48.690655, Oxford Nanopore Technologies-ONT, Oxford, UK, used May-September 2017) only showed sequence reads for which the num_genus_taxid is 1. The num_genus_taxid represents the total number of different genera out of the top three BLAST classification results. When the num_genus_taxid is 2 or 3, two or three genera are identified in the top 3 , respectively, the read is not classified at genus level but at family level (Carnobacteriaceae for the genus Dolosigranulum), in the EPI2ME report. When we looked at the EPI2ME CSV output file, we noticed that most reads (>95\%) with a Dolosigranulum genus taxID had a num_genus_taxid of 2 . When we 
added the reads with a num_genus_taxid of 2 to our results (for each genus, dashed lines in Figure 1b), the presence and abundance of Dolosigranulum sp. and also Haemophilus spp. and Ornithobacterium spp. in the nanopore versus the Illumina dataset appeared much more similar (Figure 1a,b).

For nanopore: Moraxella spp., Dolosigranulum sp. and Haemophilus spp. were most prevalent and could be detected with an abundance of at least $1 \%$ in 42,38 and 32 out of 57 samples respectively. Overall, Moraxella spp. (33\%) were most abundant, followed by Dolosigranulum sp. (18\%) and Haemophilus spp. (18\%). To compare the two sequencing platforms, the sum of the percentage of matching genera (sum of agreement) was calculated for each sample (Figure 1c). The highest sum of agreement was $96.9 \%$, the lowest $31.4 \%$, and the median was $69.1 \%$.

To assess the agreement per sample for the six main genera, Bland-Altman plots were generated. With mean differences between 0.9 and -6.0, the detection of Dolosigranulum sp., Moraxella spp., Haemophilus spp., Staphylococcus spp., and Streptococcus spp. showed good agreement between the two technologies used (Figure 2). However, Corynebacterium spp. were detected far more frequently using Illumina sequencing compared to nanopore sequencing (mean difference $=17.1$ ).

To further assess the variability between the Illumina and nanopore sequencing platforms, principal coordinate analysis and PERMANOVA statistics were performed (Supplementary Figure S2) on the microbiota profiles shown in Figure 1a,b. The platforms only contributed 5.6\% to the variations in taxonomy (Illumine versus nanopore), indicating that the platforms perform comparably.
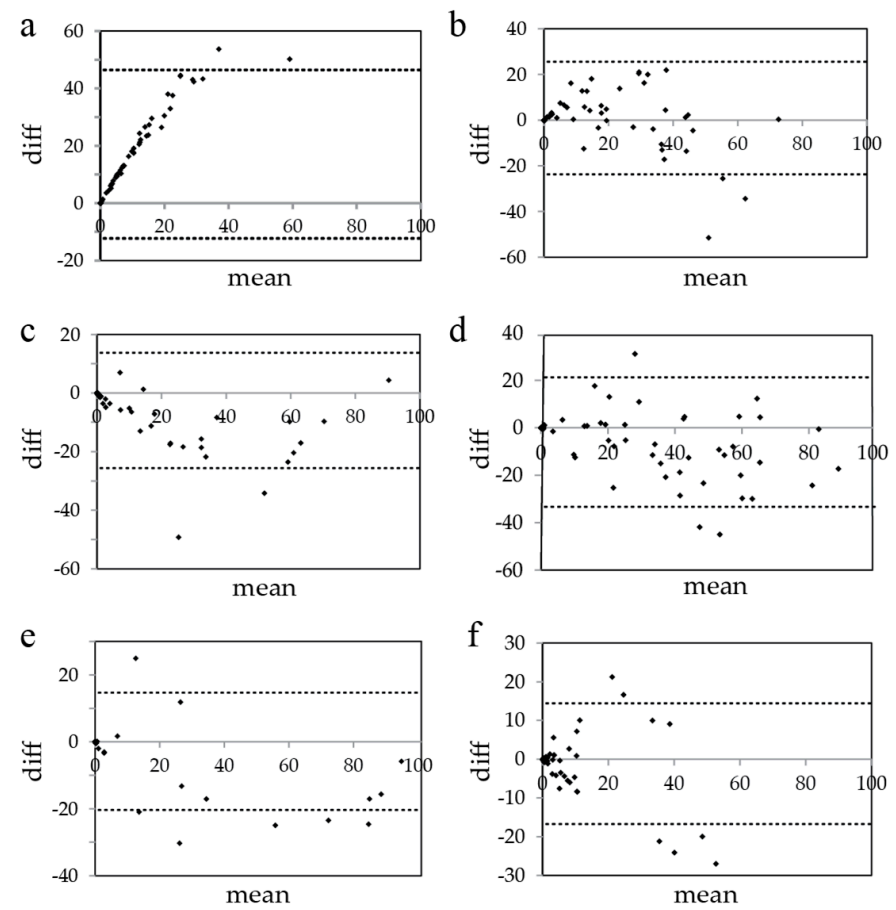

\begin{tabular}{lcc}
$\mathrm{g}$ Genus & Mean difference & Limits of agreement \\
\hline Corynebacterium & 17.1 & $-12.3,46.4$ \\
Dolosigranulum & 0.9 & $-23.8,25.5$ \\
Haemophilus & -5.9 & $-25.6,13.8$ \\
Moraxella & -6.0 & $-33.3,21.2$ \\
Staphylococcus & -2.9 & $-20.4,16.6$ \\
Streptococcus & -1.1 & $-16.7,14.4$ \\
\hline
\end{tabular}

Figure 2. Bland-Altman plots of six main genera present in the nasal microbiota. Bland-Altman plots were generated for the six main genera: (a) Corynebacterium, (b) Dolosigranulum, (c) Haemophilus, (d) Moraxella, (e) Staphylococcus, and (f) Streptococcus. For each genus, the mean difference between the two sequence methods (Illumina versus nanopore) and the limits of agreement ( $95 \%$ reference interval) were calculated and shown (g). 
In 2/7 and 6/7 (Illumina and nanopore, respectively) of the negative control samples, bacterial genera were identified (Table 1). Mostly, these genera, which included Escherichia-Shigella, Delphia and Pseudomonas (data not shown), were uncommon in nasal swabs. An exception was negative control C-6 in which $63 \%$ of the classified reads, 1500 reads in total, obtained through Illumina sequencing, were identified as Corynebacterium spp. In comparison, no reads were generated from the negative control C-6 when using nanopore sequencing.

Compared to the nose swab samples, the number of reads in the negative control samples was maximum $2.7 \%$ of the average number or raw reads of 57 samples tested and, therefore, may not have influenced the results obtained from the nasal swabs.

\subsection{Prevalence of Corynebacterium spp.}

A striking difference was the significantly lower prevalence and abundance of Corynebacterium spp. in the nanopore sequenced samples compared to the samples sequenced by Illumina technology (prevalence based on an abundance of at least 1\% per sample: $22 / 57,39 \%$ vs. $46 / 57,81 \%, p<0.001$, Chi squared test; total abundance in the combined nose swab samples: $2.2 \%$ vs $19.1 \%, p<0.001$, t-test). There was no obvious explanation for this low prevalence in the EPI2ME CSV files. When we checked whether the ONT 16S rRNA gene primes had a good match with the 16S rRNA gene of Corynebacterium spp., using the 16S rRNA gene NCBI database, we found that this was not always the case. Corynebacterium spp. that are common residents in the human nose include C. accolens, C. amycolatum, C. aurimucosum, C. propinquum, C. pseudodiphtheriticum, and C. tuberculostearicum [30,31]. Of these species, both the forward and the reverse primer were not compatible with the 16S rRNA gene of C. amycolatum, and there was only an eight basepair stretch (bp 2-9) of the forward primer that annealed to $16 \mathrm{~S}$ rRNA gene of $C$. propinquum. Thus, the $16 \mathrm{~S}$ rRNA gene will not be amplified during the PCR using the ONT 16S rRNA gene primers for the Corynebacterium species: C. amycolatum and C. propinquum. Furthermore, the first four bp ( $5^{\prime}$ end $)$ of the reversed primer could not anneal to the $16 \mathrm{~S}$ rRNA gene of C. pseudodiphtheriticum and C. tuberculostearicum. To assess how well the ONT 16S rRNA primers performed in amplifying the 16S rRNA gene, a PCR was done using DNA isolated from pure cultures of five Corynebacterium species that we had available in our hospital strain collection (C. accolens, C. amycolatum, C. diphtheria, C. pseudodiphtheriticum and C. striatum) and four species commonly present in the nasal microbiota (M. catarrhalis. H. influenzae, S. aureus, and S. pneumoniae). In agreement with the observed underrepresentation of Corynebacterium species in the samples sequenced with the Oxford Nanopore technology, we found that the 16S rRNA gene of the Corynebacterium species was poorly amplified (Figure 3).

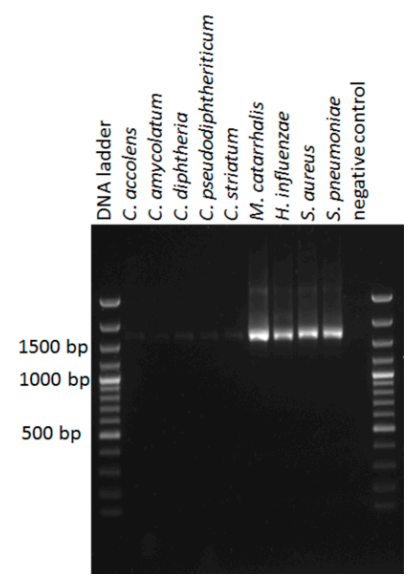

Figure 3. Agarose gel with $16 \mathrm{~S}$ rRNA gene amplicons. Total DNA was isolated from pure bacterial cultures in a similar manner as the isolation of DNA from the nasal swab samples; the DNA concentration was determined by picogreen and a PCR was performed as described for nanopore sequencing using equal amounts of template DNA, with the exception that 30 PCR cycli instead of 25 cycli were used. 


\subsection{Re-Basecalling and Analysis of the Nose Swab Samples}

To determine whether upgrades in the basecaller and the 16S EPI2ME 16S pipeline improved the detection of genera with an assigned num_genus_taxid of 2, we re-basecalled and re-analyzed the raw reads of all nose swab samples sequenced with the Oxford Nanopore technology. For this, the most recent version of the Guppy basecaller (version 3.2.10, Oxford Nanopore Technologies-ONT, Oxford, UK) and the most recent version of EPI2ME (version 2020.2.10, used April 2020, Oxford Nanopore Technologies-ONT, Oxford, UK) were used.

Instead of the num_genus_taxid, newer versions of the EPI2ME $16 \mathrm{~S}$ pipeline assign a lowest common ancestor (lca) score of 0 or 1 to the reads in the CSV file. Reads with an lca score of 0 in the newer EPI2ME version are similar to reads with a num_genus_taxid of 1 in the older version, and, by default, are considered to be accurate.

Re-basecalling slightly improved the identification of Dolosigranulum sp. (Supplementary Figure S3). However, still $81 \%$ of the reads had an lca score of 1 and were only identified at family level as Carnobacteriaceae. No improvement was observed for the identification of Haemophilus spp., of which $28 \%$ was identified at family level as Pasteurellaceae compared $30 \%$ in the initial analysis. Based on the highest scoring BLAST identification (top rank), sequence reads that were identified as Carnobacteriaceae and Pasteurellaceae did belong to the genera Dolosigranulum and Haemophilus, respectively.

\subsection{Genus and Species Level Taxonomy on Pure Cultured Single Species Bacteria Using Nanopore Sequencing}

To further evaluate how accurately nanopore sequencing of the nasal microbiota performed at genus, and also species level, we sequenced five pure culture bacterial ATCC strains that reflect species that are common to the nasal microbiota. We again followed the development of nanopore data analysis in time and sequenced the ATCC strains twice using flowcell versions R9.2 and R9.4. At genus level, $93.1-99.5 \%$ or the sequence reads were accurately identified for $4 / 5$ single species using a R9.2 flowcell and Albacore basecalling. Re-basecalling of the same sequence reads, using Guppy, showed an improvement to $97.0-99.7 \%$ accurate identification (Figure 4a). As already observed during sequencing of the nasal microbiota, poor genus identification was found for $H$. influenzae ( $55.1 \%$, R9.2 flowcell, Albacore, Figure 4a). However, upon re-basecalling using Guppy or re-sequencing using a more recent R9.4 flowcell together with Guppy basecalling, accurate identification of H. influenzae at genus level significantly improved to $89.6 \%$ in both cases.

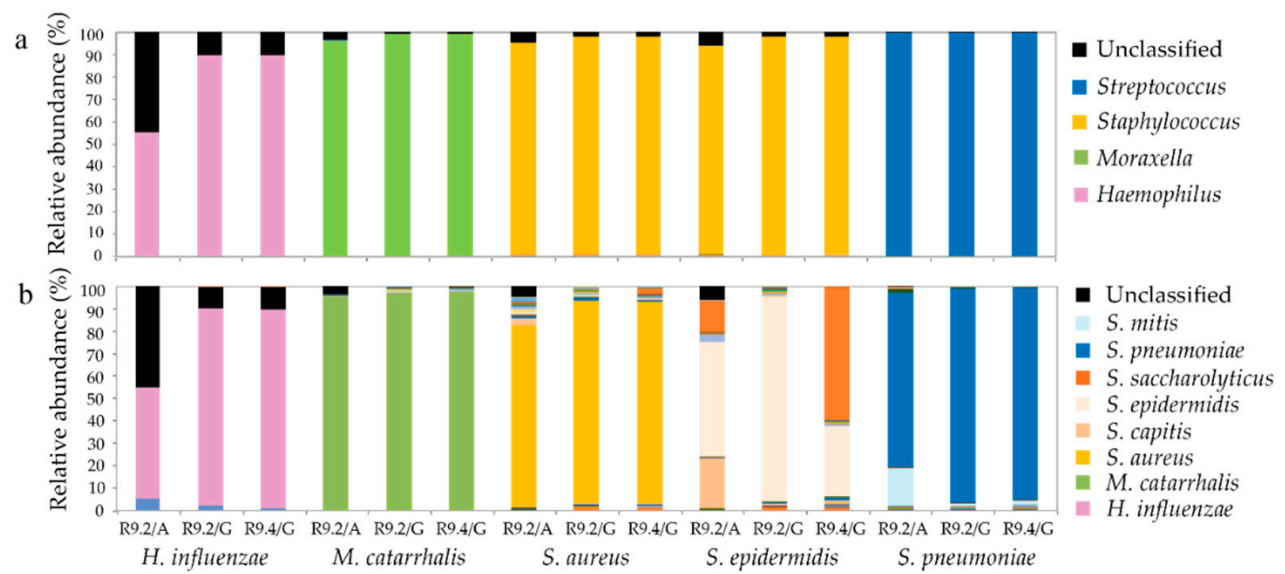

Figure 4. Genus and species level identification on pure culture species. Pure cultures of bacterial ATCC strains were sequenced using an R9.2 or R9.4 nanopore flowcell and Albacore or Guppy basecalling. Taxonomic assignment was performed at genus (a) and species (b) level using the EPI2ME 16S pipeline and the following thresholds: read length $\geq 1400 \mathrm{bp} \leq 1700 \mathrm{bp}$, num_genus_taxid is 1 or lca is 0 and accuracy $\geq 80 \%$, QC $\geq 7$ when albacore basecalling was used, or accuracy $\geq 85 \%$, QC score $\geq 9$ when Guppy basecalling was used. Similar criteria and the highest scoring BLAST identification (top rank) was used for species level identification. A is Albacore; G is Guppy basecalling. 
At species level, a similar trend of improvement was observed upon re-basecalling sequence reads, generated with a R9.2 flowcell, using Guppy, or using a R9.4 flowcell and Guppy basecalling. An exception was S. epidemidis, that, un-expectantly, showed poorer identification with the R9.4compared to the R9.2 flowcell, with $58.9 \%$ of the sequence reads being mis-identified as S. saccharolyticus (Figure $4 b$ ).

\section{Discussion}

In this study, we compared and evaluated two $16 \mathrm{~S}$ ribosomal gene sequencing strategies based on Illumina and nanopore technologies by analyzing the nasal microbiota composition of 59 human nose swab samples. In general, both sequencing techniques performed comparably at genus level except for the detection of Corynebacterium spp., a main and established genus in the nasal microbiota that was poorly detected by the Oxford Nanopore platform. New releases of a basecaller and of the nanopore flowcell led to improved genus and species identification but not for all species tested.

Upon comparing Illumina versus nanopore sequencing of the nasal microbiota samples tested, a comparable average diversity of 4.4 and 4.5 bacterial genera (Illumina versus nanopore) was detected per sample. The ISI-a measure of diversity that takes the number as well as the relative abundance of species in an environment into account-indicated greater bacterial genus diversity when Illumina sequencing was compared to nanopore, on average 2.7 versus 2.2 respectively. These numbers are lower than a previously published ISI of 4.1 for the nasal microbiota [30]. This difference may have been the result of the fact that we calculated our values based on genera instead of using operational taxonomic units (OTUs), which are more diverse and normally used for Illumina sequencing. The relative young age of the individuals sampled in the current study and the fact that many were sampled during active infection may also have resulted in our relatively low ISI values [32].

The most dominant genera detected by the Illumina platform were: Corynebacterium, Dolosigranulum, Haemophilus, Moraxella, Staphylococcus, and Streptococcus. Previous culture- and next generation sequence approaches have revealed that these are well established genera in the nasal microbiota [31].

Initially, most of the nanopore sequenced reads derived from bacteria with the genus Dolosigranulum were identified at family level only i.e., Carnobacteriaceae, which appeared to be due to fixed cut-off restrictions in the output of the Oxford Nanopore Technologies EPI2ME 16S workflow.

In the EPI2ME $16 \mathrm{~S}$ workflow, basecalled nanopore sequence reads are blasted against the NCBI $16 \mathrm{~S}$ rRNA gene database. Although it is possible that certain species are not represented in the NCBI database, this was not the case for Dolosigranulum sp. as 16S rRNA gene sequences of at least two strains are present (taxid 29394 and 883103). However, exactly because there were only two $16 \mathrm{~S}$ rRNA gene sequences of Dolosigranulum sp. present in the NCBI database, the condition of a top three blast hit with similar genera (num_genus_taxid is 1, or lca is 0 ), which is a requirement for reads to be classified using the EPI2ME $16 \mathrm{~S}$ workflow, cannot be met. Thus, the limited number of two Dolosigranulum 16S rRNA genes in the NCBI 16S rRNA gene database is probably why the EPI2ME workflow failed to identify this genus. Besides Dolosigranulum sp., the bacterial genera Haemophilus and Ornithobacterium were also identified more abundantly when read with a top three blast hit with two similar genera (num_genus_taxid is 2) together with reads with a top three blast hit with three similar genera (num_genus_taxid is 1 ) were included in the analysis. It did not become clear to us why this was the case.

When taking into account the inclusion of sequence reads with a num_genus_taxid of 1 or 2, comparison of the two sequencing platforms resulted in a median sum of agreement of $69.1 \%$, with the main genera Dolosigranulum, Moraxella, Haemophilus, Staphylococcus, and Streptococcus showing good agreement. Corynebacterium, however, was severely underrepresented in the taxonomy data generated after analysis of the nanopore sequencing results, even when reads with a num_genus_taxid other than 1 were included. Blast analysis established that two Corynebacterium species, C. amycolatum and C. propinquum, known to be habitants of the nasal microbiota [31], could not be detected due to 
potential incompatibility of the nanopore $16 \mathrm{~S}$ rRNA gene sequence primers. In agreement with this analysis, we observed that the $16 \mathrm{~S}$ rRNA gene of several pure culture Corynebacterium spp. could not be amplified using the nanopore $16 \mathrm{~S}$ primes. Incomplete annealing at the first four $5^{\prime}$ base pairs of the nanopore reverse primers, applicable for C. pseudodiphtheriticum and C. tuberculostearicum, may additionally have resulted in a low prevalence of Corynebacterium species. However, the first four $5^{\prime}$ base pairs of this reverse primer also did not match several other species that were detected in high abundance (including M. catarrhalis and M. nonliquefaciens), which tends to negate the hypothesis that poor annealing of the nanopore reverse primer led to an underrepresentation of $C$. pseudodiphtheriticum and C. tuberculostearicum. Still, mismatching of the nanopore primers and poor amplification of the $16 \mathrm{~S}$ rRNA gene of another bacterial genus, Bifidobacterium, has been reported [33]. A PCR bias due to the relatively high genomic GC-content may be another explanation why the genus Corynebacterium was underrepresented in the samples sequenced using Oxford Nanopore technology [34]. With respect to nasal microbiota profiling, our results indicate that researchers should take into account the fact that different sequencing platforms and pipelines may generate different results. However, it is usually (due to cost) not feasible to perform research microbiota profiling using multiple sequencing platforms. It should also be noted that Illumina and nanopore sequencing technologies are constantly evolving and improvements in available sequencing hardware and software platforms are constantly being made.

In this respect, we also compared taxonomic analysis performance using pure cultured bacterial isolates and the newest ONT hardware and sequence basecalling platform (R9.4 flowcells and Guppy). At genus level, we found that at least $93 \%$ of the reads were accurately identified for $4 / 5$ ATCC strains tested with a R9.2 flowcell, and an improvement for the remaining strain when we used Guppy instead of Albacore basecalling software or a R9.4 compared to a R9.2 flowcell.

Bacterial taxonomic identification at species level can be of clinical importance, as it can help guide antibiotic prescription in cases of infection, or potentially identify (prophylactic) species that suppress nasal colonization of opportunistic pathogens. For example, previous studies have demonstrated that S. epidermidis may secrete a serine protease (Esp) that is able to inhibit nasal colonization by S. aureus [35]. Further, S. mitis has been negatively associated with nasal colonization by methicillin-resistant $S$. aureus (MRSA)-apparently being able to inhibit the growth of MRSA by a hydrogen peroxide-mediated mechanism [36].

When we addressed species level identification of nanopore sequence reads, we found that $4 / 5$ pure culture species were accurately identified when using a R9.4 flowcell and Guppy basecalling. However, species identification of S. epidermidus was found to occur with almost $60 \%$ of reads being mis-classified as $S$. saccharolyticus. This mis-classification may have been the result of a high degree of sequence similarity between the S. epidemidis and S. saccharolyticus $16 \mathrm{~S}$ rRNA gene. Because the bacteria were grown under aerobic conditions in which anaerobic S. saccharolyticus does not grow, contamination of the S. epidermidus culture with S. saccharolyticus before DNA isolation is not plausible.

\section{Conclusions}

In conclusion, the current study shows that microbiota profiling of the human nasal microbiota, using nanopore sequencing platform, is comparable to Illumina sequencing at the genus level and above. However, nanopore sequencing may not accurately identify bacteria within the genus Corynebacterium. At the species level, it appears that advances still need to be made to improve the accuracy of taxonomic classification by nanopore sequencing (as with other sequencing technologies). Since our initial comparative studies began, accurate taxonomic assignment at species level using nanopore sequencing continues to improve, with advances in reducing the relatively high error rate of nanopore sequencing, generating obvious advantages. Such changes are to be welcomed. However, constantly evolving hardware and software outputs complicate downstream data analysis and make the comparison of historically published results with more recent results potentially problematic. 
Ethics Approval and Consent to Participate: Approval for the sampling protocol (protocol version 4, date 08-08-2014) was obtained prior to study start from the Medical Ethical Committee of University Medical Centre Utrecht (14-104, approval date: 09-09-2014) and the Institutional Review Boards of Hillel Yaffe Medical Centre (HYMC-0108-13 and HYMC-0107-13), Bnai Zion Medical Centre (BNZ-0107-14 and BNZ-0011-14) and Hadassah University Medical Centre (HMO-0007-14 and HMO-0006-14). Written, informed consent was obtained from each patient-participant by research staff (by research nurse, research fellow or the principal investigator) prior to enrolment in the study.

Supplementary Materials: The following are available online at http:/www.mdpi.com/2073-4425/11/9/1105/s1. Figure S1: Rarefaction curves of sequenced nasal swab samples, Figure S2: Principle coordinate analysis on Illumina and nanopore nasal microbiota profiles. Figure S3: Re-basecalling of nanopore sequence reads derived from nasal swabs.

Author Contributions: Sample collection, C.B.v.H. and L.J.B.; DNA isolation D.H.-K. and A.P.H.; Illumina sequencing S.A.B. and R.K.; Nanopore sequencing A.P.H. and D.H.-K.; software development, data analysis and data curation, A.P.H., S.A.B., R.J., S.D.H., A.P.S., and W.d.K.; statistical analysis, A.P.H., R.K. and M.A.J.d.R.; writing-original draft preparation, A.P.H. and J.P.H.; review and editing, all authors; funding acquisition, A.P.S., L.J.B. and J.P.H.; supervision, A.P.S. and J.P.H. All authors have read and agreed to the published version of the manuscript.

Funding: This work received funding from the European Union's Seventh Framework Programme for Health under grant agreement number 602860 (TAILORED-Treatment; www.tailored-treatment.eu).

Acknowledgments: We thank A.C., E.E. and K.O., MeMed, Tirat Carmel, Israel and Dan Engelhard, Hadassah Medical Centre, Ein Kerem, Israel, for their contribution to collecting nose swab samples; and D.F. and E.G., NorayBio, Bilbao, Spain for help with microbiota database management. All authors have read and agreed to the published version of the manuscript.

Conflicts of Interest: The authors declare no conflict of interest.

\section{References}

1. Mansbach, J.M.; Luna, P.N.; Shaw, C.A.; Hasegawa, K.; Petrosino, J.F.; Piedra, P.A.; Sullivan, A.F.; Espinola, J.A.; Stewart, C.J.; Camargo, C.A., Jr. Increased Moraxella and Streptococcus species abundance after severe bronchiolitis is associated with recurrent wheezing. J. Allergy Clin. Immunol. 2020, 145, 518-527.e8. [CrossRef]

2. Bomar, L.; Brugger, S.D.; Lemon, K.P. Bacterial microbiota of the nasal passages across the span of human life. Curr. Opin. Microbiol. 2017, 41, 8-14. [CrossRef]

3. Mika, M.; Korten, I.; Qi, W.; Regamey, N.; Frey, U.; Casaulta, C.; Latzin, P.; Hilty, M.; SCILD Study Group. The nasal microbiota in infants with cystic fibrosis in the first year of life: A prospective cohort study. Lancet Respir. Med. 2016, 4, 627-635. [CrossRef]

4. Hui, J.W.; Ong, J.; Herdegen, J.J.; Kim, H.; Codispoti, C.D.; Kalantari, V.; Tobin, M.C.; Schleimer, R.P.; Batra, P.S.; LoSavio, P.S.; et al. Risk of obstructive sleep apnea in African American patients with chronic rhinosinusitis. Ann. Allergy Asthma Immunol. 2017, 118, 685-688.e1. [CrossRef]

5. Shah, D.; Ajami, N.J.; Ghantoji, S.S.; Shelburne, S.; El_Haddad, D.; Shah, P.; Piedra, P.; Shpall, E.; Kontoyiannis, D.P.; Chemaly, R.F. Nasal Microbiota Changes are Associated with Progression to Lower Respiratory Infection Following Respiratory Syncytial Virus Upper Respiratory Infection in Hematopoietic Cell Transplant Recipients. Open Forum Infect. Dis. 2016, 3, 2232. [CrossRef]

6. Man, W.H.; de Steenhuijsen Piters, W.A.; Bogaert, D. The microbiota of the respiratory tract: Gatekeeper to respiratory health. Nat. Rev. Microbiol. 2017, 15, 259-270. [CrossRef]

7. Man, W.H.; van Houten, M.A.; Mérelle, M.E.; Vlieger, A.M.; Chu, M.L.J.N.; Jansen, N.J.G.; Sanders, E.A.M.; Bogaert, D. Bacterial and viral respiratory tract microbiota and host characteristics in children with lower respiratory tract infections: A matched case-control study. Lancet Respir. Med. 2019, 7, 417-426. [CrossRef]

8. Lu, Y.J.; Sasaki, T.; Kuwahara-Arai, K.; Uehara, Y.; Hiramatsu, K. Development of new application for comprehensive viability analysis based on microbiome analysis by next-generation sequencing: Insights into staphylococcal carriage in human nasal cavities. Appl. Environ. Microbiol. 2018, 84. [CrossRef]

9. Sadowsky, M.J.; Staley, C.; Heiner, C.; Hall, R.; Kelly, C.R.; Brandt, L.; Khoruts, A. Analysis of gut microbiota-An ever changing landscape. Gut Microbes 2017, 8, 268-275. [CrossRef]

10. Rohde, H.; Burandt, E.C.; Siemssen, N.; Frommelt, L.; Burdelski, C.; Wurster, S.; Scherpe, S.; Davies, A.P.; Harris, L.G.; Horstkotte, M.A.; et al. Polysaccharide intercellular adhesin or protein factors in biofilm accumulation of Staphylococcus epidermidis and Staphylococcus aureus isolated from prosthetic hip and knee joint infections. Biomaterials 2007, 28, 1711-1720. [CrossRef] 
11. Oxford Nanopore Technologies. Available online: https://nanoporetech.com/ (accessed on 1 June 2020).

12. Shin, J.; Lee, S.; Go, M.J.; Lee, S.Y.; Kim, S.C.; Lee, C.H.; Cho, B.K. Analysis of the mouse gut microbiome using full-length 16S rRNA amplicon sequencing. Sci. Rep. 2016, 6, 29681. [CrossRef]

13. Cusco, A.; Vines, J.; D'Andreano, S.; Riva, F.; Casellas, J.; Sanchez, A.; Francino, O. Using MinION'TM to characterize dog skin microbiota through full-length 16S rRNA gene sequencing approach. bioRxiv 2017, 167015. [CrossRef]

14. Mitsuhashi, S.; Kryukov, K.; Nakagawa, S.; Takeuchi, J.S.; Shiraishi, Y.; Asano, K.; Imanishi, T. A portable system for rapid bacterial composition analysis using a nanopore-based sequencer and laptop computer. Sci. Rep. 2017, 7, 5657. [CrossRef]

15. Laver, T.; Harrison, J.; O’Neill, P.A.; Moore, K.; Farbos, A.; Paszkiewicz, K.; Studholme, D.J. Assessing the performance of the Oxford Nanopore Technologies MinION. Biomol. Detect. Quantif. 2015, 3, 1-8. [CrossRef]

16. Lee, A.S.; de Lencastre, H.; Garau, J.; Kluytmans, J.; Malhotra-Kumar, S.; Peschel, A.; Harbarth, S. Methicillin-resistant Staphylococcus aureus. Nat. Rev. Dis. Primers 2018, 4, 18033. [CrossRef] [PubMed]

17. Van Houten, C.B.; Oved, K.; Eden, E.; Cohen, A.; Engelhard, D.; Boers, S.; Kraaij, R.; Karlsson, R.; Fernandez, D.; Gonzalez, E.; et al. Observational multi-centre, prospective study to characterize novel pathogen-and host-related factors in hospitalized patients with lower respiratory tract infections and/or sepsis-the "TAILORED-Treatment" study. BMC Infect. Dis. 2018, 18, 377. [CrossRef] [PubMed]

18. Heikema, A.; de Koning, W.; Li, Y.; Stubbs, A.; Hays, J.P. Lessons learnt from the introduction of nanopore sequencing? Clin. Microbiol. Infect. 2020. [CrossRef]

19. Yang, S.; Lin, S.; Kelen, G.D.; Quinn, T.C.; Dick, J.D.; Gaydos, C.A.; Rothman, R.E. Quantitative multiprobe PCR assay for simultaneous detection and identification to species level of bacterial pathogens. J. Clin. Microbiol. 2002, 40, 3449-3454. [CrossRef]

20. Bogaert, D.; Keijser, B.; Huse, S.; Rossen, J.; Veenhoven, R.; van Gils, E.; Bruin, J.; Montijn, R.; Bonten, M.; Sanders, E. Variability and diversity of nasopharyngeal microbiota in children: A metagenomic analysis. PLoS ONE 2011, 6, e17035. [CrossRef]

21. Fadrosh, D.W.; Ma, B.; Gajer, P.; Sengamalay, N.; Ott, S.; Brotman, R.M.; Ravel, J. An improved dual-indexing approach for multiplexed 16S rRNA gene sequencing on the Illumina MiSeq platform. Microbiome 2014, 2, 6. [CrossRef]

22. Schloss, P.D.; Westcott, S.L.; Ryabin, T.; Hall, J.R.; Hartmann, M.; Hollister, E.B.; Lesniewski, R.A.; Oakley, B.B.; Parks, D.H.; Robinson, C.J.; et al. Introducing mothur: Open-source, platform-independent, community-supported software for describing and comparing microbial communities. Appl. Environ. Microbiol. 2009, 75, 7537-7541. [CrossRef] [PubMed]

23. Batut, B.; Gravouil, K.; Defois, C.; Hiltemann, S.; Brugere, J.F.; Peyretaillade, E.; Peyret, P. ASaiM: A Galaxy-based framework to analyze microbiota data. Gigascience 2018, 7, giy057. [CrossRef] [PubMed]

24. Pruesse, E.; Quast, C.; Knittel, K.; Fuchs, B.M.; Ludwig, W.; Peplies, J.; Glockner, F.O. SILVA: A comprehensive online resource for quality checked and aligned ribosomal RNA sequence data compatible with ARB. Nucleic Acids Res. 2007, 35, 7188-7196. [CrossRef] [PubMed]

25. Silva Reference Files. Available online: https://www.Mothur.org/wiki/Silva_reference_files (accessed on 29 August 2020).

26. Oxford Nanopore Technologies EPI2ME. Available online: https://epi2me.nanoporetech.com/ (accessed on 29 August 2020).

27. NCBI BLAST. Available online: https://blast.ncbi.nlm.nih.gov/Blast.cgi?PAGE_TYPE=BlastSearch\&BLAST_ SPEC=TargLociBlast (accessed on 29 August 2020).

28. Python Library ete2. Available online: https://pypi.org/project/ete2/ (accessed on 29 August 2020).

29. European Nucleotide Archive (ENA). Available online: https://www.ebi.ac.uk/ena/data/search?query= PRJEB28612 (accessed on 11 September 2020).

30. De Boeck, I.; Wittouck, S.; Wuyts, S.; Oerlemans, E.F.M.; van den Broek, M.F.L.; Vandenheuvel, D.; Vanderveken, O.; Lebeer, S. Comparing the Healthy Nose and Nasopharynx Microbiota Reveals Continuity As Well As Niche-Specificity. Front. Microbiol. 2017, 8, 2372. [CrossRef]

31. Brugger, S.D.; Bomar, L.; Lemon, K.P. Commensal-Pathogen Interactions along the Human Nasal Passages. PLoS Pathog. 2016, 12, e1005633. [CrossRef]

32. Biswas, K.; Hoggard, M.; Jain, R.; Taylor, M.W.; Douglas, R.G. The nasal microbiota in health and disease: Variation within and between subjects. Front. Microbiol. 2015, 9, 134. [CrossRef] 
33. Kai, S.; Matsuo, Y.; Nakagawa, S.; Kryukov, K.; Matsukawa, S.; Tanaka, H.; Iwai, T.; Imanishi, T.; Hirota, K. Rapid bacterial identification by direct PCR amplification of $16 \mathrm{~S}$ rRNA genes using the MinION nanopore sequencer. FEBS Open Bio 2019, 9, 548-557. [CrossRef]

34. Laursen, M.F.; Dalgaard, M.D.; Bahl, M.I. Genomic GC-Content Affects the Accuracy of 16S rRNA Gene Sequencing Based Microbial Profiling due to PCR Bias. Front. Microbiol. 2017, 8, 1934. [CrossRef]

35. Iwase, T.; Uehara, Y.; Shinji, H.; Tajima, A.; Seo, H.; Takada, K.; Agata, T.; Mizunoe, Y. Staphylococcus epidermidis Esp inhibits Staphylococcus aureus biofilm formation and nasal colonization. Nature 2010, 465, 346-349. [CrossRef]

36. Bessesen, M.T.; Kotter, C.V.; Wagner, B.D.; Adams, J.C.; Kingery, S.; Benoit, J.B.; Robertson, C.E.; Janoff, E.N.; Frank, D.N. MRSA colonization and the nasal microbiome in adults at high risk of colonization and infection. J. Infect. 2015, 71, 649-657. [CrossRef]

(C) 2020 by the authors. Licensee MDPI, Basel, Switzerland. This article is an open access article distributed under the terms and conditions of the Creative Commons Attribution (CC BY) license (http://creativecommons.org/licenses/by/4.0/). 